\title{
Clinical Study to Evaluate the Value of CT Urography in Hematuria
}

\author{
Sunil Kumar Pusthey ${ }^{1}$, Raju Ragidi², Nagaraju Baja ${ }^{3}$ \\ ${ }^{1}$ Assistant Professor, ${ }^{2}$ Assistant Professor, ${ }^{3}$ Senior Resident Department of Radiology, Osmania Medical College, Koti, \\ Hyderabad, Telangana, India.
}

Corresponding author: Dr. Raju Ragidi, Assistant Professor, Department of Radiology, Osmania Medical College, Koti, Hyderabad, Telangana, India.

DOI: http://dx.doi.org/10.21276/ijcmsr.2020.5.1.26

BY-NC-ND

How to cite this article: Sunil Kumar Pusthey, Raju Ragidi, Nagaraju Baja. Clinical study to evaluate the value of CT urography in hematuria. International Journal of Contemporary Medicine Surgery and Radiology. 2020;5(1):A115-A122.

\section{A B S T R A C T}

Introduction: Haematuria is a sign that can arise from a constellation of disorders, ranging from urinary tract infection to invasive malignancy. Study aimed to evaluate the value of CT urography in hematuria.

Material and methods: In present study, 58 patients presented with hematuria (both microscopic and macroscopic) were evaluated with CT urography.

Results: In present study, most common age group presented with hematuria were between 31-45 years and constitute about $41 \%$ of total number of cases. In this study there was male (40) preponderance than females(18). Most common cause of hematuria in present study was urolithiasis contributing to $40 \%$ of total cases, most common location of urolithiasis being ureteric. Most common age group for urolithiasis was below 50 years. $2^{\text {nd }}$ most common cause for hematuria in the present study includes urinary tract neoplasms, out of these bladder carcinoma (50\% of total neoplasms) was the main cause for hematuria in present study group.

Conclusion: CT Urography showed high diagnostic values with a combination of unenhanced, nephrographic phase and excretory phases.

Keywords: Clinical Study, CT, Urography, Hematuria

\section{INTRODUCTION}

Hematuria is one of the most common manifestations of urinary tract disease. Hematuria can originate from any site along the urinary tract and has a wide range of causes, including calculi, neoplasm, infection, trauma, coagulopathy, and renal parenchymal disease. This makes the differential diagnosis extensive and seemingly disjointed The concept of CT Urography (CTU) is more appropriate as both the renal parenchyma and urothelium can be evaluated with one relatively non invasive comprehensive examination. ${ }^{1}$ The rationale for CT urography is that patients with hematuria can be fully investigated by a single imaging technique with a high degree of sensitivity and specificity. It is especially suitable for patients presenting with hematuria where the urinary tract must be assessed for stone disease and neoplasms of the kidney and/or urothelium. If there are only a small number of red blood cells in the urine, the urine color might not change and the blood cells are only apparent under a microscope. This is usually referred to a microscopic hematuria. The prevalence of microscopic hematuria in asymptomatic individuals is approximately 2.5\% Larger amounts of blood in the urine can cause a discoloration of the urine from a light pinkish hue to a dark red or even brown appearance. This is known as gross hematuria or macroscopic hematuria. Multidetector CT urography (MDCTU) is a single examination that allows evaluation of potential urinary tract calculi, renal parenchymal masses, and both benign and malignant urothelial lesions.

With the advent of mluti-detector row CT scanners, evaluation of the urothelium of the entire urinary tract with high-resolution thin sections during a single breath- hold has become a reality. Initial results with this new technique are encouraging. Current investigations of MDCTU focus on methods to improve opacification and distension of the upper urinary tract-the collecting systems, pelvis, and ureters. The role of abdominal compression, infusion of saline and/or furosemide, and optimal time delay of excretory phase imaging is being explore. Upper tract urothelial malignancies, including small lesions less the $5 \mathrm{~mm}$ in diameter, can be detected with high sensitivity. Intravenous urography, ultrasonography, CT, retrograde ureterography and pyelography, cystoscopy, and ureteroscopy can all be used to evaluate patients with hematuria. The main advantage of the technique is its ability to detect uroepithelial malignancies. CT urography provides a detailed anatomic depiction of each of the major portions of the urinary tract-the kidneys, intrarenal collecting systems, ureters and bladder and thus allows patients with hematuria 
to be evaluated comprehensively.

This technique is based on the acquisition of non enhanced and enhanced CT scans of the abdomen and pelvis, including the essential acquisition of thin section helical CT scans of the urinary tract during the excretory phase of enhancement. Multiplanar 2 dimensional and 3 dimensional reformation images are produced from axial source images during the excretory phase. ${ }^{2} \mathrm{CT}$ urography offers several advantages for imaging of the urinary tract: single breath- hold coverage of the entire urinary tract with absence of respiratory misregistration, rapid imaging with optimum contrast medium opacification and reduced partial volume effect as appropriate slices can be selected from the volumetric data. ${ }^{3}$ Study aimed to evaluate the value of CT urography in hematuria.

\section{MATERIAL AND METHODS}

It was prospective study of 2 years from October 2016 to September 2018 in Department of Radio diagnosis, Mamata Medical College, Khammam. 58 patients with history of hematuria (both microscopic and macroscopic), between 10-75 years of age will be subjected to study which includes out patients, in patients, referral patients of Mamata general hospital, Urology department, Khammam.

Inclusion Criteria: Patients presenting with hematuria (both microscopic and macroscopic).

Exclusion Criteria: Patients below 10 and above 75 years of age, pregnant and lactating patient, severe renal failure, previous allergic reaction to contrast media 27 . The patients will undergo a 3 phase CT examination after obtaining informed consent in written form.

First phase is the initial non-contrast phase. Second phase is the nephrographic phase, which will be acquired following a delay of 90- 100 seconds after administration of $100-120 \mathrm{ml}$ of intravenous iodinated contrast, to evaluate the renal parenchyma.

Followed by the pyelographic phase which will be taken $8-10$ minutes following administration, to evaluate the urothelium from the pelvicaliceal system to the bladder. Equipment: This will be performed with a 16slice Multidetector row CT scanner (Siemens somatom scope). Scans will be obtained from the kidneys to the bladder in a craniocaudal fashion, with the following scanning parameters as slice thickness$5 \mathrm{~mm}$, increment thickness $-1.2 \mathrm{~mm}$, pitch-1.5, mas- $25, \mathrm{Kv}-$ 130 and Collimater fov-300 $\mathrm{mm}$.

Three-dimensional (3D) reconstructions of the non enhanced, nephrogenic phase and excretory phase will be performed. The follow up diagnosis will be established on the basis of histopatholigic findings or the findings at a urologic procedure (cystoscopy, ureteroscopy and

Unenhanced coronal and nephrographic phase axial images showing left ureterocele with two calculi in it. Ureterocele was later confirmed with ureteroscopy.

Nephrographic coronal and pyelographic coronal images show illdefined heterogenously hyper enhancing mass lesion noted in lower pole of left kidney, the lesion shows contrast washout in excretory pyelographic phase- suggestive of renal cell carcinoma. Later the case confirmed as renal cell carcinoma on histopathological examination (fig-2,3).

\section{RESULTS}

Most common age group for hematuria was 31-45 years which constituted $41 \%$ of the study group and least common age group was $61-75$ years constitutes $16 \%$. prevalence of hematuria was more common among males(40) compared to females(18) (table-1).

Prevalence of painless hematuria(33) was more common than painful hematuria(25). Macroscopic hematuria constituted

\begin{tabular}{|l|c|c|}
\hline Age in intervals & $\begin{array}{c}\text { No of patients } \\
(\mathbf{n = 5 8})\end{array}$ & Percentage \\
\hline 15-30yrs & 14 & 24 \\
\hline 31-45yrs & 24 & 41 \\
\hline 46-60yrs & 11 & 19 \\
\hline $61-75 y r s$ & 9 & 16 \\
\hline Gender & & 69 \\
\hline Males & 40 & 31 \\
\hline Females & 18 & \\
\hline \multicolumn{2}{|r|}{} \\
\hline
\end{tabular}

Table-1: Distribution of patients according to age

\begin{tabular}{|l|c|c|}
\hline \multicolumn{4}{|l|}{ Pain among patients with hematuria } \\
\hline Painless & 33 & 57 \\
\hline Painful & 25 & 43 \\
\hline patients according to nature of hematuria \\
\hline Microscopic hematuria & 31 & 54 \\
\hline Macroscopic hematuria & 27 & 46 \\
\hline \multicolumn{3}{|l|}{ Table-2: Pain among patients with hematuria in present study } \\
\hline
\end{tabular}

\begin{tabular}{|l|c|c|}
\hline Cause of hematuria & $\begin{array}{c}\text { No of } \\
\text { patients } \\
\text { (n=58) }\end{array}$ & Percentages \\
\hline Urolithiasis & 23 & 39.6 \\
\hline Renal cell carcinoma & 3 & 5.2 \\
\hline Angiomyolipoma & 1 & 1.7 \\
\hline Pyelonephritis & 2 & 3.4 \\
\hline Renal abscess & 2 & 3.4 \\
\hline Normal & 5 & 8.6 \\
\hline Renal complex cysts & 5 & 8.6 \\
\hline Urinoma & 2 & 3.4 \\
\hline PCKD & 1 & 1.7 \\
\hline Vesico-vaginal fistula & 1 & 1.7 \\
\hline Ectopic kidney & 1 & 1.7 \\
\hline Renal hydatid & 1 & 1.7 \\
\hline Urogenital TB & 1 & 1.7 \\
\hline $\begin{array}{l}\text { Xanthogranulomatous } \\
\text { pyelonephritis }\end{array}$ & 1 & 1.7 \\
\hline Intra peritoneal bladder rupture & 1 & 1.7 \\
\hline Bladder carcinoma & 4 & 6.8 \\
\hline Cystitis & 3 & 5.1 \\
\hline Horseshoe kidney & 1 & 1.7 \\
\hline \multicolumn{2}{|c|}{ Table-3: Distribution of patients according to cause of } \\
\hline \multicolumn{2}{|l|}{ hematuria } & \\
\hline
\end{tabular}




\begin{tabular}{|l|c|c|}
\hline Location of calculus & $\begin{array}{c}\text { No of } \\
\text { patients }(\mathbf{n}=\mathbf{2 3})\end{array}$ & Percentages \\
\hline Renal & 5 & 21.7 \\
\hline PUJ & 4 & 17.4 \\
\hline Ureteric & 8 & 34.8 \\
\hline VUJ & 6 & 26.1 \\
\hline Cause of hydronephrosis & & \\
\hline Renal calculus & 2 & 8.7 \\
\hline PUJ calculus & 4 & 17.4 \\
\hline Ureteric calculus & 8 & 34.7 \\
\hline VUJ calculus & 6 & 26.1 \\
\hline Bladder carcinoma & 2 & 8.7 \\
\hline PUJ obstruction & 1 & 4.3 \\
\hline Urogenital TB & 1 & 4.3 \\
\hline \multicolumn{2}{|c|}{ Table-4: Distribution of patients according to location of } \\
\multicolumn{2}{|c|}{ calculus } \\
\hline
\end{tabular}

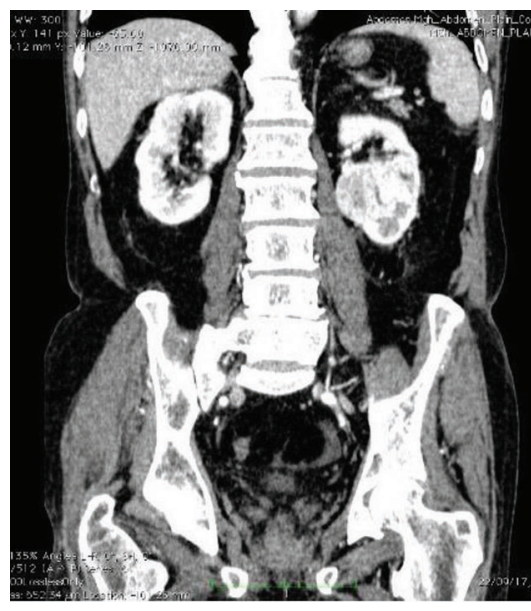

Figure-2: Nephrographic coronal

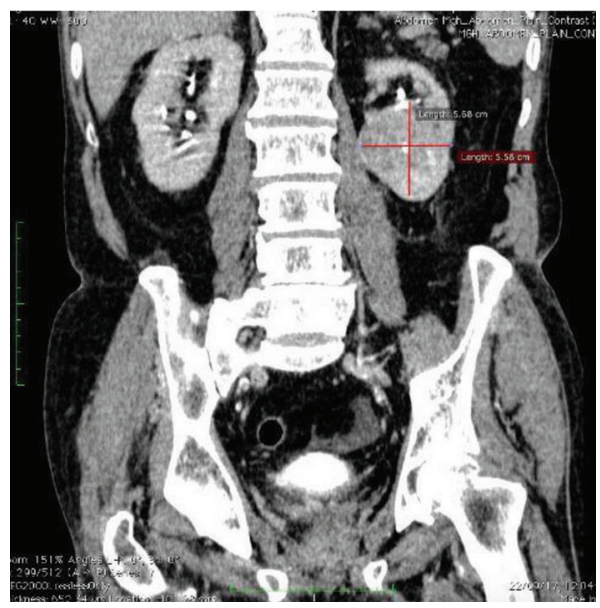

Figure-3: Pyelographic coronal

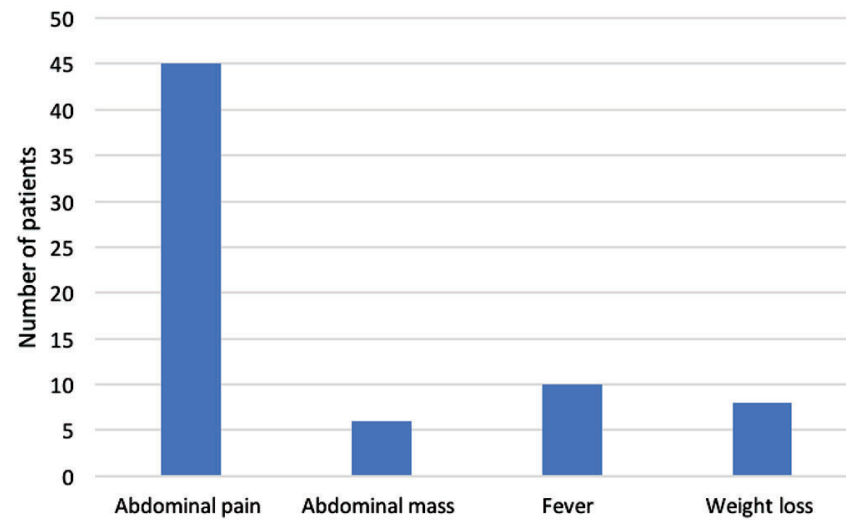

Figure-4: Clinical manifestations other than hematuria

CT Urography detects 23 patients having urolithiasis as the cause for hematuria including renal, PUJ, ureteric and VUJ calculi and which constitutes to nearly about $40 \%$ in the total number of cases an thus becomes the main cause of hematuria in the present study (table-4).

Most of the urolithiasis patients present with painful microscopic hematuria and in present study most common location of urolithiasis was ureteric (35\%) followed by VUJ (figure-5).

Most common age group having hematuria were below 50 years constituting nearly about 70\% (of total urolithiasis 


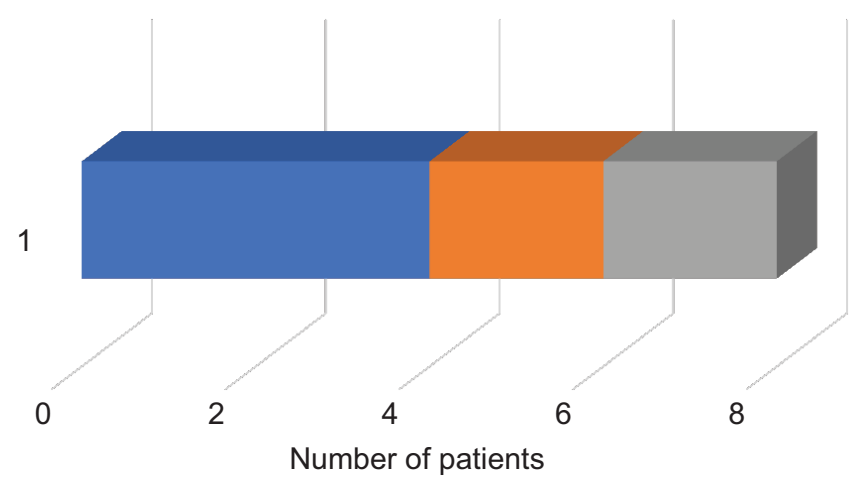

Renal Only Vesical Vesical+ Ureteric extension

Figure-5: Distribution of neoplasms according to location along urinary tract.

patients) though the below 50 years age group dominates with number, of patients there was no clear significance between the two as $p$ value comes nearly about 0.92 . $(\mathrm{P}<0.05$ is significant) (table-5).

Most of the cases of neoplasms in present study were above 50 years and the table depicts relationship between age group and neoplasms as significant with $\mathrm{p}$ value between them around is 0.01 which was less than 0.05 .

\section{DISCUSSION}

Hematuria is one of the most common manifestation of urinary tract diseases. It can originate from any site along the urinary tract and has spectrum of causes including calculi, neoplasm, infection, trauma, medications, coagulopathy and renal parenchymal diseases. Assessment of urological malignancies are probably the most important reason for evaluating these patients to get an early and accurate diagnosis. Therefore, examinations with a high sensitivity for the detection of neoplasms are essential. The ability to detect other possible causes of hematuria is also important.

Hematuria can be well evaluated with a comprehensive contrast material- enhanced multi- detector row computed tomography (CT) protocol that combines unenhanced, nephrographic-phase, and excretory phase imaging. Unenhanced images are obtained from the kidneys to the bladder and allow optimal detection of renal calculi, a common cause of hematuria. Renal parenchymal abnormalities, particularly masses, are best visualized on nephrographic-phase images.

Thin-section delayed images obtained from the kidneys to the bladder demonstrate the urinary tract distended with contrast material and are useful in detecting urothelial disease. The ability of MDCT urography to evaluate both the renal parenchyma as well as urothelium in a single investigation has prompted many authors to moot it as potential one stop investigation for the spectrum of urinary tract disorders presenting with hematuria.

Present study comprised of 58 patients who presented with hematuria (both macroscopic and microscopic) and were referred to radiology department from urology department for work up with CT urography. All the patients underwent
$\mathrm{CT}$ urography and diagnosis was established after analyzing all the phases of study with required post processing techniques.

Among these 58 patients, 5 patients with lower urinary tract pathologies also underwent flexible cystoscopy and the results were compared with the CT urography findings. In this study the most common age group for hematuria was 31-45 years which constituted $41 \%$ of the study group and least common age group was $61-75$ years constitutes $16 \%$. In this study, prevalence of hematuria was more common among males(40) compared to females(18). (Table-1)

In this study, prevalence of painless hematuria (33) was more common than painful hematuria(25). Macroscopic hematuria constitutes (54\%) which was more than microscopic hematuria. Abdominal pain was the most common complaint other than hematuria followed by fever. 58 patients the cause of hematuria was divided in to urolithiasis, infective, inflammatory, congenital, traumatic, neoplasms and other miscellaneous conditions.

\section{Urolithiasis}

In the present study, CT Urography detects 23 patients having urolithiasis as the cause for hematuria including renal, puj, ureteric and vuj calculi and which constitutes to nearly about $40 \%$ in the total number of cases an thus becomes the main cause of hematuria in the present study.

Similar study, role of Computerized Tomographic Urography in the Initial Evaluation of Hematuria by Albani JM et al $(2007)^{4}$, studied source of hematuria in 107 patients and concluded the most common cause of hematuria as urolithiasis(26\%). Most of the urolithiasis patients present with painful microscopic hematuria and in present study most common location of urolithiasis was ureteric $(35 \%)$ followed by vuj. Similar study on role of Multi-detector row computed tomography urography (MDCTU) in the evaluation of microscopic hematuria in adults by Mahmoud MA et al (2015) $)^{5}$, described urolithiasis as the cause for microscopic hematuria in about $24 \%$ of patients.

In the present study, most common age group having hematuria were below 50 years constituting nearly about $70 \%$ (of total urolithiasis patients) though the below 50 years age group dominates with number, of patients there was no clear significance between the two as $\mathrm{p}$ value comes nearly about 0.92. ( $\mathrm{P}<0.05$ is significant $)$.

The age group correlation shown above thus signifies that higher number of patients below 50 years occurred by chance. All of these calculi were identified on the unenhanced CT images. CT urography had no false-negative results, which means no calculi was detected either surgically or by other imaging techniques after a negative CT urogram. On CTU other than detection of calculi, many other clinically relevant observations were also made which helps in management. Signs of obstruction like perinephric fat stranding, periureteric edema, hydroureter, hydronephrosis and lateral conal fascial thickening were clearly depicted. Most of the urolithiasis patients also show hydronephrosis, most common cause being ureteric calculus. 
A male patient of age 30 years presented with hematuria and CT urography reaveals presence of cystocele with VUJ calculus and thus concluded VUJ calculus 53 as cause of hematuria and ureterocele was confirmed by ureteroscopy and thus psedoureterocele of VUJ calculus was ruled out.

Another female patient aged 35 years presented with hematuria on CT urogram revealed left ectopic kidney with left ureteric calculus and mild hydronephrosis of left ectopic kidney and the cause for hematuria concluded to be ureteric calculus rather than ectopic kidney.

In a similar study Kumar S et al $(2011)^{6}$, described a similar case with ectopic kidney presenting with hematuria also revealed pelvic calculus with hydronephrosis which was found to be cause of hematuria evaluated through contrast enhanced computed tomography.

\section{Infective}

In present study there were various infective causes along with its complications noted affecting both upper and lower urinary tract, includes pyelonephritis, renal abscess, renal hydatid, urogenital tuberculosis, and cystitis. In this study infective causes constitutes to about $16 \%$ and most of them present with painful hematuria along with most common symptom other than hematuria includes fever. Acute pyelonephritis is usually well characterized by MDCTU with findings of a "striated nephrogram" in a swollen kidney and stranding of the perinephric fat. Occasionally there can be thickening of the pelvicaliceal wall, which can also show increased mucosal enhancement. ${ }^{7}$ In acute pyelonephritis, MDCTU typically demonstrates solitary or multifocal hypodense areas with obliteration of normal corticomedullary differentiation. Parenchymal abnormalities are best demonstrated on CT images obtained in the nephrographic phase while excretory phase acquisitions are better for diagnosing renal abscesses than images acquired in the corticomedullary or nephrographic phases ${ }^{8}$. The nephropyelographic phase of the two-phase protocol combines the advantages of both these phases in a single acquisition.

In present study there were 2 cases of acute pyelonephritis, one case was 28 year old male patient presented with fever and right flank pain along with painless microscopic hematuria on ct urogram showed enlarged right kidney with perinephric fat stranding and in nephrogenic phase showed striated nephrogram. Another case was 38 year old female presented with pelvic pain and fever along with hematuria showed ectopic kidney with its focal enlargement and bulging on left side with various hypo densities with in cortex noted in nephrogenic phase along with right kidney showing similar findings with perinephric fat stranding.

In a similar study by Tsao YT et al $(2008)^{9}$,described a similar case of ectopic kidney with focal pyelonephtitis as cause of hematuria which was confirmed by contrast enhanced computed tomography. Two cases of renal abscess were present which were well delineate in excretory phase as fluid density focal areas with perinephric fat stranding. 45 year old male patient present with microscopic painless hematuria on ct urography showed well defined multi locular cystic lesion with mixed density and enhancing septa and wall noted in left kidney. The above case suspected to be renal hydatid cyst and was confirmed as same on surgical exploration.

Ishimitsu DN et al $(2010)^{10}$, described a similar case presented with microscopic hematuria, left flank mass and hydatiduria concluded that contrast adminsterd CT necessary to know the enhancement of septa and wall. Gharde P et al(2012) $)^{11}$, reported a similar case of 40 year old male patient presented with intermediate hematuria and hydatiduria since 10 years. On CT revealed multiseptated cystic lesion which on surgery confirmed as renal hydatid.

In present study another case, female of 22 years presented with fever since 2 months, weight loss and on urine examination few red blood cells noted and the case further investigated with ct urography which revealed thimble shaped bladder with circumferential wall thickening and decreased capacity noted along with multiple ureteric strictures and mild bilateral hydronephrosis.

The case was given provisional diagnosis as urogenital tuberculosis involving bladder and ureter and the case was confirmed as urogenital tb by cystoscopic biopsy sample and treated accordingly. Figueiredo AA et al $(2008)^{12}$, described few patients presented with hematuria on urine examination along with other constitutional symptoms of tuberculosis and they described the bladder involvement with most of hematuria patients. Altintepe $\mathrm{L}$ et al $(2005)^{13}$, depicted about $35 \%$ of urogenital tuberculosis patients having contracted thimble bladder on imaging and described about $12 \%$ of patients presented with macroscopic hematuria.

Present study showed that hematuria may not be presenting complaint of patient with urogenital tuberculosis but it can be later discovered during further investigations. About 3 patients presented with hematuria(includes both microscopic and macroscopic), they were further evaluated with ct urography revealed, 2 cases showing diffuse thickening of bladder wall and one case showing focal thickening of bladder wall in its posterior aspect.

The first two cases were diagnosed as cystitis and treated accordingly and patient relieved with the symptoms. Third case suspected to be bladder carcinoma and biopsy was taken under cystoscopic guidance, later the biopsy sample proved to be of infective cause and then managed accordingly and this case was described as false positive for bladder carcinoma on ct urography.

O'malley $\mathrm{ME}$ et al $(2003)^{14}$, similar study conducted on patients presenting with painless hematuria in sample of 91 patients by ct urography, about two patients confirmed of cystitis. Shinagare AB et al $(2011)^{15}$, described many infective and inflammatory causes of bladder such as cystitis will simulate as bladder carcinoma on ct urography and concluded that they should be confirmed by cystoscopic biopsy and proceed for further management accordingly.

\section{Inflammatory causes}

In this study, a female patient aged 38 years presented with painful microscopic hematuria advised for $\mathrm{x}$ ray KUB, noted staghorn calculus in left kidney and pelvic calculus in right 
kidney then the case further investigated with ct urography, revealed similar findings along with delayed nephrogenic contrast enhancement of cortex of right kidney compared to left kidney and right kidney show persistent delayed contrast enhancement even in pyelographic delayed scans.

Right kidney also showed hypoattenuating dilated calyces with abrupt blunting of pelvis with a pelvic calculus giving bear paw appearance. The case was suspected as xanthogranulomatous pyelonephritis and then urology team advised radionuclide scan to know functioning status of right kidney and concluded it as non functional kidney and proceeded for nephrectomy and sent to pathology. Histopathology confirmed the xanthogranulomatous changes thus in present study ct urography could accurately diagnose a case of xanthogranulomatous pyelonephritis.

Although sensitivity and specificity could not be commented with a single case of present study but ct urography is useful to know associated findings in xanthogranulomatous disease such as staghorn and pelvic calculi, abrupt narrowing at pelvis causing dilation of calyceal system and extrarenal manifestations.

Nataluk EA et al $(1995)^{16}$, reviewed 12 patients with a clinicopathogenic diagnosis of xanthogranulomatous pyelonephritis (XGP) and to determine if a computed tomography $(\mathrm{CT})$ scan is the imaging procedure of choice for diagnosis. Nine of 10 patients $(90 \%)$ who were evaluated by CT scan had the correct diagnosis made prior to nephrectomy and concluded CT has proven to be the most accurate imaging study to evaluate xanthogranulomatous pyelonephritis. Gupta S et $\mathrm{al}(2010)^{17}$, discussed a case of female patient with similar findings to present study that 17-year-old female who presented with a 2-month history of increasing abdominal pain and intermittent episodes of increased frequency and dysuria. Plain antero-posterior radiograph of the abdomen revealed a left staghorn calculus. Computed tomography scan with intravenous contrast revealed a low-density inflammatory area and reduced cortical dye uptake on the left renal parenchyma as compared to the opposite side. A dimercapto-succinic acid renal scan revealed that the affected kidney contributed $18 \%$ of differential function. A diagnosis of XPN was made.

Gupta S et $\mathrm{al}(2010)^{17}$ tried to manage the patient conservatively with percutaneous nephrostomy tube placement but the process become unsuccessful, finally they did nephrectomy and diagnosis was confirmed.

\section{Congenital}

In present study, a case of 15 year old female patient presented with microscopic painless hematuria on ct urography evaluation, cause of hematuria found to be left ectopic kidney with renal calculus, both acting synergistically as cause for hematuria in the present case. In a similar study by Bhoil $\mathrm{R}$ et $\mathrm{al}(2015)^{18}$, described a similar case of ectopic kidney with renal calculus as source of hematuria. In present study, another male patient of 50 years presented with painless hematuria revealed horseshoe kidney with renal calculus on ct urography as cause of hematuria.

\section{Trauma}

In present study, male patient aged 32 years had trauma and presented with painless macroscopic hematuria underwent ct urography revealed contrast extravasation into peritoneal cavity in pyelographic delayed scans and diagnosed as a case of intraperitoneal bladder rupture. Nephrogenic phase helpful in diagnosing upper urinary tract injuries mostly renal injuries and pyelographic phase helpful in diagnosing lower urinary tract injuries mostly bladder injuries. In a similar study by Ramchandani $\mathrm{P}$ et $\mathrm{al}(2009)^{19}$, described role of ct urography along with other imaging modalities in genitourinary trauma and depicted that ct urography helpful in revealing both upper and lower urinary tract.

\section{Upper urinary tract neoplasms}

Renal neoplasms: In present study, a total of 4 renal neoplasms were identified as cause of hematuria out of which 3 were detected to be malignant, 1 case was benign neoplasm. In this study, 3 cases of renal cell carcinoma, each case was diagnosed as clear cell subtype with one case showing cystic changes within it. In malignant cases the predominant age group in present study was above 50 years and almost all the cases presented with painless macroscopic hematuria along with abdominal mass. One case of renal cell carcinoma showed liver metastasis and another case of RCC revealed presence of renal vein thrombus. In the present study, a 46 years female patient presented with painless microscopic hematuria, on ct urography revealed angiomyolipoma as cause of hematuria. In the present study, upper urinary tract neoplasms contribute to about nearly $7 \%$ of all cases presented with hematuria.

CT urography had no false-negative or false positive results, which means no patient was detected to have renal neoplasm either by other imaging techniques or follow up after a negative CT urogram. Many important observations were made in evaluation of renal malignancies like Nephrographic phase optimally depicted lesions due to background homogenous enhancement of renal parenchyma. In this study, corticomedullary phase by-passed with no loss of any clinically relevant findings there by reducing the total radiation dose. Renal vascular invasion, infiltration into collecting system, distortion of calyces were all clearly depicted with high sensitivity.

\section{Lower urinary tract neoplasms}

Bladder carcinoma found to be major cause of hematuria in lower urinary tract neoplasms in the present study. All the cases of lower urinary tract neoplasms presented with macroscopic painless hematuria. Lower urinary tract neoplasms were about $7 \%$ of all cases, that is about 4 cases. All the 4 cases are bladder carcinomas, out of these two cases showed vuj and ureteric extension and two cases limited to bladder itself. All the cases of lower urinary tract neoplasm in present study were of transitional cell type of carcinomas. A case of focal cystitis was false positively diagnosed as bladder carcinoma in present study, later on cystoscopic biopsy confirmed as cystitis. In the present study, CT urography had a sensitivity of $100 \%$ and positive predictive value of $80 \%$. 
In a similar study, Blick CG et al $(2012)^{20}$, concluded that there was a clear advantage for the diagnostic strategy using $\mathrm{CT}$ urography and flexible cystoscopy as a triage test for rigid cystoscopy and follow-up, in which patients with a positive CT urography score for bladder cancer were directly referred for rigid cystoscopy, but all other patients undergo flexible cystoscopy.

\section{All neoplasms both upper and lower urinary tract}

In present study, second most common cause of hematuria are urinary tract neoplasms contributing to about $14 \%$ of cases that is about 8 cases in total combining both benign and malignant causes. In present study, most of the neoplasms of urinary tract presented above 50 years age group constituting about $75 \%$.

Distribution of patients with neoplasm according to age group $\mathrm{P}<0.05$ is significant, that most of the cases of neoplasms in present study were above 50 years and the table depicts relationship between age group and neoplasms as significant with $p$ value between them around is 0.01 which is less than 0.05 . Significant $p$ value shows that neoplasms in above 50 years age group occurred not by chance.

\section{Miscellaneous}

In this study, a male patient aged 32 years presented with microscopic painless hematuria. Ct urography revealed multiple simple and complex cysts in both kidneys with no involvement of other organs and diagnosis of bilateral polycystic kidney disease was made. The cause of hematuria in present case was bilateral polycystic kidney disease. In a similar study by Hateboer N(1999) ${ }^{21}$, described polycystic kidney disease manifests with hematuria in advanced stages of disease.

In present study, about 5 cases presenting with microscopic hematuria were confirmed on ct urography as having complex cysts in their kidneys. About $8 \%$ of hematuria patients were having complex cysts as single best cause for hematuria in present study. In present study, about $8 \%$ of patients with hematuria on ct urography revealed no cause and they concluded as normal cases.

In this study, 2 cases with hematuria on ct urography revealed urinomas as cause of hematuria. In this study a case of post hysterectomy patient presented with complaints of hematuria, on ct urography revealed vesico vaginal fistula as cause of hematuria. In present study as shown in table 8 in results, hydronephrosis seen in about 24 cases, most common cause being urolithiasis followed by bladder carcinoma.

Urolithiasis contribute to $80 \%$ of total number of cases with hydronephrosis and ureteric location of calculus being the most common cause within urolithiasis. Diagnostic accuracy of CT Urography in evaluating the etiology of hematuria: In present study group of 58 patients, all the patients having upper urinary tract pathologies were diagnosed accurately with CT urography. There were no false negatives or false positive results among those patients of upper urinary tract pathologies.

\section{CONCLUSION}

Unenhanced phase of ct urography is useful in detecting urolithiasis, nephrographic phase mostly useful for upper urinary tract pathologies and pyelographic phases are useful for detecting lower urinary tract pathologies. According to present study, CT urography had more diagnostic accuracy in detecting upper urinary tract pathologies than lower urinary tract pathologies. Lower urinary tract pathologies especially bladder carcinoma, cystoscopy had better diagnostic accuracy than CT urography.

However CT urography gives a good roadmap in evaluating patients suspected having lower urinary tract pathologies before performing a cystoscopy and combining both would yield a high diagnostic outcome. According to present study, CT Urography showed high diagnostic values with a combination of unenhanced, nephrographic phase and excretory phases.

\section{REFERENCE}

1. Owan NC, Turney BW, Taylor NJ, McCarthy CL, Crew JP. Multidetector computed tomography urography for diagnosing upper urinary tract urothelial tumour. BJU international. 2007;99(6):1363-70.

2. Jinzaki M, Tanimoto A, Shinmoto H, Horiguchi Y, Sato K, Kuribayashi S, Silverman SG. Detection of bladder tumors with dynamic contrast- enhanced MDCT. American Journal of Roentgenology. 2007;188(4):9138.

3. Maher MM, Kalra MK, Rizzo S, Mueller PR, Saini S. Multidetector CT urography in imaging of the urinary tract in patients with hematuria. Korean journal of radiology. 2004;5(1):1-0.

4. Albani JM, Ciaschini MW, Streem SB, Herts BR, Angermeier KW. The role of computerized tomographic urography in the initial evaluation of hematuria. The Journal of urology. 2007;177(2):644-8.

5. Mahmoud MA, Mahmoud MZ, Omer MA, Garalnabi ME, Abukonna A, Fagiri MA. Multi-Detector Row Computed Tomography Urography (MDCTU) in the Evaluation of Microscopic Hematuria in Adults. Open Journal of Radiology. 2015;5(01):20.

6. Kumar S, Bolla SR, Vollala VR. Unilateral ectopic kidney in the pelvis-a case report. Chang Gung Med J. 2011;34(6 Suppl):10-2.

7. Kawashima A, LeRoy AJ. Radiologic evaluation of patients with renal infections. Infectious Disease Clinics. 2003;17(2):433-56.

8. Kawashima A, Sandler CM, Goldman SM. Current roles and controversies in the imaging evaluation of acute renal infection. World journal of urology. 1998;16(1):9-17.

9. Tsao YT, Lin SH, Lin YF, Chu P. Pelvic ectopic kidney with acute pyelonephritis: wolf in sheep's clothing. The American journal of emergency medicine. 2008;26(4):517-e3.

10. Shimitsu DN, Saouaf R, Kallman C, Balzer BL. Renal hydatid disease. Radiographics. 2010;30(2):334-7.

11. Gharde P, Wagh DD, Patil A. Left renal hydatid cyst presenting as hematuria and macroscopic hydatiduria 
since last ten years. Tropical parasitology. 2012;2(1):58.

12. Figueiredo AA, Lucon AM. Urogenital tuberculosis: update and review of 8961 cases from the world literature. Reviews in urology. 2008;10(3):207.

13. Altintepe L, Tonbul HZ, Ozbey I, Guney I, Odabas AR, Cetinkaya R, Piskin MM, Selcuk Y. Urinary tuberculosis: ten years' experience. Renal failure. 2005;27(6):657-61.

14. O'malley ME, Hahn PF, Yoder IC, Gazelle GS, McGovern FJ, Mueller PR. Comparison of excretory phase, helical computed tomography with intravenous urography in patients with painless haematuria. Clinical radiology. 2003;58(4):294-300.

15. Shinagare AB, Sadow CA, Sahni VA, Silverman SG. Urinary bladder: normal appearance and mimics of malignancy at CT urography. Cancer Imaging. 2011;11(1):100.

16. Nataluk EA, McCullough DL, Scharling EO. Xanthogranulomatous pyelonephritis, the gatekeeper's dilemma: a contemporary look at an old problem. Urology. 1995;45(3):377-80.

17. Gupta S, Araya CE, Dharnidharka VR. Xanthogranulomatous pyelonephritis in pediatric patients: case report and review of literature. Journal of pediatric urology. 2010;6(4):355-8.

18. Bhoil R, Sood D, Singh YP, Nimkar K, Shukla A. An ectopic pelvic kidney. Polish journal of radiology. 2015;80:425.

19. Ramchandani $P$, Buckler PM. Imaging of genitourinary trauma. American Journal of Roentgenology. 2009;192(6):1514-23.

20. Blick CG, Nazir SA, Mallett S, Turney BW, Onwu NN, Roberts IS, Crew JP, Cowan NC. Evaluation of diagnostic strategies for bladder cancer using computed tomography (CT) urography, flexible cystoscopy and voided urine cytology: results for 778 patients from a hospital haematuria clinic. BJU international. 2012;110(1):84-94.

21. Hateboer N, v Dijk MA, Bogdanova N, Coto E, SaggarMalik AK, San Millan JL, Torra R, Breuning M, Ravine D, European PKD1-PKD2 Study Group. Comparison of phenotypes of polycystic kidney disease types 1 and 2. The Lancet. 1999;353(9147):103-7.

Source of Support: Nil; Conflict of Interest: None

Submitted: 31-12-2019; Accepted: 24-01-2020; Published online: 22-02-2020 